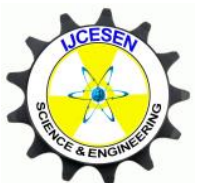

Copyright (C) IJCESEN
International Journal of Computational and

Experimental Science and $\boldsymbol{E N g i n e e r i n g}$

(IJCESEN)

Vol. 5-No.2 (2019) pp. 100-104

http://iakkurt.dergipark.gov.tr/ijcesen

Research Article

\title{
Unimodal Optimization of Axially Compressed Shear Deformable Columns
}

\author{
İzzet Ufuk ÇAĞDAŞ*
}

Akdeniz University, Engineering Faculty, Civil Engineering Department, 07058, Antalya-Turkey

* Corresponding Author : izzetufuk@gmail.com

ORCID: 0000-0002-2528-2978

\section{Article Info:}

DOI: $10.22399 /$ ijcesen.591907

Received : 15 July 2019

Accepted : 31 July 2019

\section{Keywords}

Optimal column

Shear deformation

Optimality condition

Iterative solution

\begin{abstract}
:
In this study, the unimodal optimality conditions for axially compressed shear deformable columns are formulated and the optimal design problems defined are numerically solved using an iterative procedure based on the finite element method. The results presented show that the optimization results are more reliable than the ones obtained using classical beam theory as cross-sectional area does not vanish at points where the bending moment is equal to zero.
\end{abstract}

\section{Introduction}

Starting from the differential equations given by Timoshenko [1], the optimality conditions related to the optimization problems defined are derived and a numerical technique is used to find the optimal shapes of shear deformable compressed columns. The results are in good agreement with the analytical and numerical results obtained in this study, and obtained from different sources. However, comparison can only be made for the limiting case when there's no shear deformation which corresponds to a very long column with very high shear rigidity.

Several researchers have tried to solve the strongest column problem analytically or by employing numerical techniques for different support and loading conditions [2-5]. However, the shear deformation, contribution of which becomes very important especially when the column length is short [2-6], has never been considered, even for the discrete optimization approaches. Also, this exclusion leads to thinner sections, which is important especially when optimizing statically indeterminate columns as the unimodal optimality condition leads to optimal designs where area vanishes at certain parts of the statically indeterminate columns. Also, stress has been used as a constraint in only a few papers [7-9], which becomes important when column length is short or material is weak.

The study is limited with unimodal optimization as there's enough complexity, and there's no other source to compare the results. It was stated by Ziegler [10] that, the effect of axial shortening on buckling load may be comparable in magnitude to that of the shear deformation. However, the influence of axial shortening is neglected in this study.

Finally, the numerical results obtained for some selected cases are presented in tabular and graphical form and the influence of shear deformation on the optimal designs is demonstrated.

\section{Problem Definition}

The governing differential equations (d.e.) are given below in equations (1) and (2),

$$
\frac{d}{d x}\left[C\left(\frac{d w}{d x}-\theta\right)\right]+P \frac{d^{2} w}{d x^{2}}=0
$$




$$
\frac{d}{d x}\left(D \frac{d \theta}{d x}\right)+C\left(\frac{d w}{d x}-\theta\right)=0
$$

where, $C=k G A, D=E I$ or $D=E \alpha_{n} A^{n}$ with the boundary conditions, $w(0)=w(L)=0, \theta=0$ at a clamped support, and $D d \theta / d x=0$ at a simple support.

There are two independent variables in the d.e.; $w$, deflection, and $\theta$, rotation. Thus, the weak form can be obtained by multiplying equations (1) and (2) by $w$ and by $\theta$ respectively and integrating over the length. The residuals $e_{1}$ and $e_{2}$, and their sum, $e_{t}$ are given below,

$$
\begin{aligned}
e_{1} & =\int_{0}^{L} w\left\{\frac{d}{d x}\left[C\left(\frac{d w}{d x}-\theta\right)\right]+P \frac{d^{2} w}{d x^{2}}\right\} d x \\
& =-\int_{0}^{L} C \frac{d w}{d x}\left(\frac{d w}{d x}-\theta\right) d x-P \int_{0}^{L}\left(\frac{d w}{d x}\right)^{2} d x \\
e_{2} & =\int_{0}^{L} \theta\left\{\frac{d}{d x}\left(D \frac{d \theta}{d x}\right)+C\left(\frac{d w}{d x}-\theta\right)=0\right\} d x \\
& =-\int_{0}^{L} D\left(\frac{d \theta}{d x}\right)^{2} d x+\int_{0}^{L} C \theta\left(\frac{d w}{d x}-\theta\right) d x \\
e_{t} & =e_{1}+e_{2}=0
\end{aligned}
$$

Using eq. (3), and re-arranging, the quotient giving the buckling load $P$ is calculated as given in eq. (4),

$$
P=\frac{\int_{0}^{L}\left[\alpha_{n} E A^{n}\left(\frac{d \theta}{d x}\right)^{2}+k G A\left(\frac{d w}{d x}-\theta\right)^{2}\right] d x}{\int_{0}^{L}\left(\frac{d w}{d x}\right)^{2} d x}
$$

Equation (4) can also be obtained using the Minimum Potential Energy Theorem as given in [11].

\subsection{Optimal design problems}

The minimum area and maximum stress constraints are to be studied in separate problems which are named as Problem I and Problem II. The statements of these optimal design problems are given next.

Problem I. Find the cross-sectional area $A(x)$ of the optimal column for given values of $n$ and $A_{0}$ such that the buckling load $P$ is the maximum subject to the volume, $\int_{0}^{L} A d X=V$, and minimum area,
$A \geq A_{0}$, constraints.

Problem II. Find the cross-sectional area $A(x)$ of the optimal column for given values of $n$ and $\square_{y s}$ such that the buckling load $P$ is the maximum subject to the volume $\int_{0}^{L} A d X=V$, and the maximum stress $\frac{P}{A} \leq \sigma_{y s}$ constraints.

\subsection{The unimodal optimality conditions}

The Lagrangian related to Problem I is given below,

$$
\begin{aligned}
& L_{11}=\frac{\int_{0}^{L}\left[\alpha_{n} E A^{n}\left(\frac{d \theta}{d x}\right)^{2}+k G A\left(\frac{d w}{d x}-\theta\right)^{2}\right] d x}{\int_{0}^{L}\left(\frac{d w}{d x}\right)^{2} d x}-\ldots \\
& \ldots \kappa_{1}\left(\int_{0}^{L} A d x-V\right)-\int_{0}^{1} \kappa_{2}(x)\left[-h^{2}(x)-A_{0}+A\right] d x
\end{aligned}
$$

Setting the variation of $L_{11}$ yields the following result;

$$
\begin{aligned}
& n E \alpha_{n} A^{n-1}\left(\frac{d \theta}{d x}\right)^{2}+k G\left(\frac{d w}{d x}-\theta\right)^{2} \\
& =\left(\kappa_{1}+\kappa_{2}(x)\right) \int_{0}^{L}\left(\frac{d w}{d x}\right)^{2} d x
\end{aligned}
$$

Setting the variation of $h(x)$ equal to zero reveals the fact that, $\kappa_{2}(x)=0$ when minimum area constraint is not violated, and $\kappa_{2}(x) \neq 0$ when violated. Thus, the optimality condition for problem I becomes,

$$
A= \begin{cases}{\left[\frac{n M^{2}}{C-E \alpha_{n} k G\left(\frac{d w}{d x}-\theta\right)^{2}}\right]^{1 / n+1}} & , x \in x_{a} \\ A_{0} & , x \notin x_{a}\end{cases}
$$

where $x_{a}, M$, denote the set of points where the constraint is not active, and bending moment respectively. The constant $C$ can be calculated by using the volume constraint.

The Lagrangian related to Problem II is given below, 


$$
\begin{aligned}
& L_{12}=\frac{\int_{0}^{L}\left[\alpha_{n} E A^{n}\left(\frac{d \theta}{d x}\right)^{2}+k G A\left(\frac{d w}{d x}-\theta\right)^{2}\right] d x}{\int_{0}^{L}\left(\frac{d w}{d x}\right)^{2} d x}-\ldots \\
& \ldots \kappa_{1}\left(\int_{0}^{L} A d x-V\right)-\int_{0}^{1} \kappa_{2}(x)\left[-h^{2}(x)-\sigma_{y s}+\frac{P}{A}\right] d x
\end{aligned}
$$

The optimality condition for problem II is the same as the one given in eq.(5) except that $A_{0}$.is replaced with $\frac{P}{\sigma_{y s}}$.

\section{Finite Element Stability Analysis}

A first order shear deformable beam element is used to calculate the buckling load. The detailed derivation of the stiffness matrix is given in [12], but the geometric stiffness is formulated in this section.

\subsection{Energy formulation}

The total potential energy, $\pi$, of the beam is given below in eq. (6) when the axial strain is neglected.

$$
\begin{aligned}
\pi & =\frac{1}{2} \int_{L}\left(\frac{M^{2}}{E I}+\frac{V^{2}}{\alpha G A}\right) d x-P w-\int_{L} q w d x \\
& =\frac{1}{2} \int_{L} E I\left(\frac{d \theta}{d x}\right)^{2} d x+\frac{1}{2} \int_{L} S \varphi^{2} d x-P w-\int_{L} q w d x
\end{aligned}
$$

where $L$ is the length of the beam $E I$ is the flexural stiffness, $S$ is the shear stiffness, $M(x)$ is the bending moment, $V(x)$ is the shear force, $q$ is the uniformly distributed force, $w(x)$ is the deflection, $P$ is the point load, $\theta$ is the total rotation of the midsurface, and $\phi$ is the rotation due to shear.

\subsection{Finite element formulation}

The element has 3 nodes and the unknowns at the nodes, i.e. the nodal dof, are the transverse deflection $w_{i}$, and rotation $\theta_{l}$ where $i=1,2,3$. The element displacement vector $\boldsymbol{\delta}_{e}$ contains the transverse deflections and rotations at the nodes of the element.

$$
\boldsymbol{\delta}_{e}^{T}=\left\{\begin{array}{llllll}
w_{1} & \theta_{1} & w_{2} & \theta_{2} & w_{3} & \theta_{3}
\end{array}\right\}
$$

The shape (interpolation) functions are given below,

$$
\begin{aligned}
& N_{1}(\xi)=\frac{1}{2} \xi(\xi-1), \\
& N_{2}(\xi)=(1-\xi)(1+\xi),
\end{aligned}
$$

$$
N_{3}(\xi)=\frac{1}{2} \xi(1+\xi)
$$

where $\xi$ is the local coordinate of the sampling point.

The Jacobian: It is assumed that node 2 is in the middle of the element. Then the Jacobian, $\mathbf{J}=L^{e} / 2$ where $L^{e}$ is the length of the element.

Strain-Displacement relations: Strains can be defined in terms of deflections and derivatives of interpolation function. The derivatives needed are calculated using the chain rule of differentiation and the Jacobian.

$$
\left\{\begin{array}{c}
\frac{d \theta}{d x} \\
\phi
\end{array}\right\}=\left[\begin{array}{cc}
0 & \frac{d}{d x} \\
\frac{-d}{d x} & 1
\end{array}\right]\left\{\begin{array}{l}
w \\
\theta
\end{array}\right\}
$$

Stress-strain relations: For a beam made up of homogeneous, isotropic material, the stress strain relations are given below in eq.(10).

$$
\left\{\begin{array}{l}
M \\
V
\end{array}\right\}=\left[\begin{array}{cc}
E I & 0 \\
0 & k G A
\end{array}\right]\left\{\begin{array}{l}
\theta \\
\phi
\end{array}\right\}, \mathbf{D}^{\prime}=\left[\begin{array}{cc}
E I & 0 \\
0 & k G A
\end{array}\right]
$$

\section{Calculation of the element stiffness and geometric stiffness matrices}

The linearized stability analysis can be performed using the element stiffness and geometric stiffness matrices defined below for the special case when the mid-side node is in the middle.

$$
\begin{aligned}
k^{e} & =\int_{-1}^{1} \mathbf{B}^{T} \mathbf{D}^{\prime} \mathbf{B} \frac{L^{e}}{2} d \xi \\
k_{g}^{e} & =\int_{-1}^{1} \mathbf{G}^{T} \mathbf{G} \frac{L^{e}}{2} d \xi
\end{aligned}
$$

where, the sub-matrices $\mathbf{B}$ and $\mathbf{G}$ are given below and $N_{i}^{\prime}=\frac{d N_{i}}{d \xi}$.

$$
\mathbf{B}^{\mathrm{T}}=\left[\begin{array}{cc}
0 & -2 N_{1}^{\prime} / L^{e} \\
2 N_{1}^{\prime} / L^{e} & N_{1} \\
0 & -2 N_{2}^{\prime} / L^{e} \\
2 N_{2}^{\prime} / L^{e} & N_{2} \\
0 & -2 N_{3} / L^{e} \\
2 N_{3}^{\prime} / L^{e} & N_{3}
\end{array}\right]
$$




$$
\mathbf{G}=\left[\begin{array}{llllll}
N_{1}^{\prime} & 0 & N_{2}^{\prime} & 0 & N_{3}^{\prime} & 0
\end{array}\right]
$$

\section{Optimization Procedure}

An iterative optimization procedure is used to solve the optimization problems defined. The moments and shear forces at the element nodes are calculated using the $\mathbf{D}$ and $\mathbf{B}$ matrices evaluated at the nodal points. $d w / d x$ at the nodes $1,2,3$ of the element can be obtained using the following equation,

$$
\left\{\begin{array}{c}
\frac{d w_{1}}{d x} \\
\frac{d w_{2}}{d x} \\
\frac{d w_{2}}{d x}
\end{array}\right\}=\frac{L^{e}}{2}\left[\begin{array}{cccccc}
-\frac{3}{2} & 0 & 2 & 0 & \frac{1}{2} & 0 \\
-\frac{1}{2} & 0 & 0 & 0 & \frac{1}{2} & 0 \\
\frac{1}{2} & 0 & -2 & 0 & \frac{3}{2} & 0
\end{array}\right]\left\{\begin{array}{c}
w_{1} \\
\theta_{1} \\
w_{2} \\
\theta_{2} \\
w_{3} \\
\theta_{3}
\end{array}\right\}
$$

which is valid for the case when node 2 is at the middle of the element. The averages of the derivatives shall be taken for adjacent element nodes.

The iterative scheme is defined as follows:

i) Assume a uniform area distribution. $i=0$.

ii) Compute $P$ and $d w / d x, \phi$, and $M$ at the nodes

iii) Assume a value for $C$.

iv) Calculate $A_{i}, x_{a}$ according to the optimality condition depending on the problem.

v) Calculate $C$ using the volume constraint.

vi) $\quad$ Go to Step iv until $C$ value converges.

vii) Calculate $A_{i}$ using the iteratively calculated $C$ value.

viii) Modify $A_{i}$ according to Eq. (15) below. Set $i=i+1$.

ix) $\quad$ Go to Step ii until $P$ value converges.

To increase the stability of the solution, the last calculated $A$ is modified in step viii as the weighted average of area obtained in the current iteration step and the one computed in the preceding iteration as

$$
A_{i}=\frac{\theta A_{i}+(1-\theta) A_{i-1}}{100}
$$

where $\theta \in[0,1]$, and its value is taken as 0.98 which was found to be an optimal value. Failure to implement Eq. (15) in the iterative process leads to an unstable iterative scheme.

\section{Numerical Results}

A column of length $L$ and height $h$ is discretized using 51 finite elements and the material properties are taken as $E=2 \times 10^{6}, v=0.3$. Here only Problem I is solved with $A_{0}=0$. The efficiencies of a simply supported column under compression are given below in Table 1 in comparison with the optimal Euler columns, where,

$\eta(\%)=\frac{P_{o p t}-P_{0}}{P_{0}} \times 100, \lambda=\frac{L}{h}$

and $P_{o p t}$, and $P_{0}$ are the optimal buckling load, and the buckling load of the uniform column respectively.

It can be observed from Table 1 that, the efficiencies are lower for lower $\lambda$ values because the influence of shear deformation on buckling is higher for shorter columns. Also, the decrease in efficiency percentage increases with increasing $n$. However, the efficiencies do not considerably differ for the cases where $\lambda \geq 25$.

Table 1. Optimization results for the simply supported column

\begin{tabular}{|c|c|c|c|c|c|}
\hline$n$ & $\begin{array}{c}\eta \\
(\text { Euler, } \\
\lambda=\infty)\end{array}$ & $\begin{array}{c}\eta \\
(\lambda=100)\end{array}$ & $\begin{array}{c}\eta \\
(\lambda=50)\end{array}$ & $(\lambda=25)$ & $\begin{array}{c}\eta \\
(\lambda=10)\end{array}$ \\
\hline 1 & 21.6 & 21.30 & 21.01 & 20.08 & 16.12 \\
\hline 2 & 33.3 & 32.67 & 32.49 & 31.79 & 27.71 \\
\hline 3 & 41 & 39.56 & 39.44 & 38.87 & 35.23 \\
\hline
\end{tabular}

The optimal distributions of area for $n=1,2,3$ and the same input variables, i.e. $\lambda=10 A_{0}=0.0$ and $\sigma_{y s}=\infty$, are plotted below in Figures 1(a), 1(b), and 1 (c) respectively.

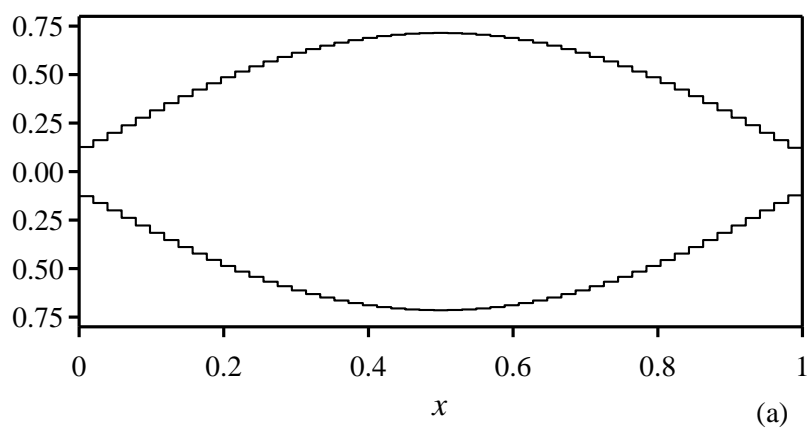



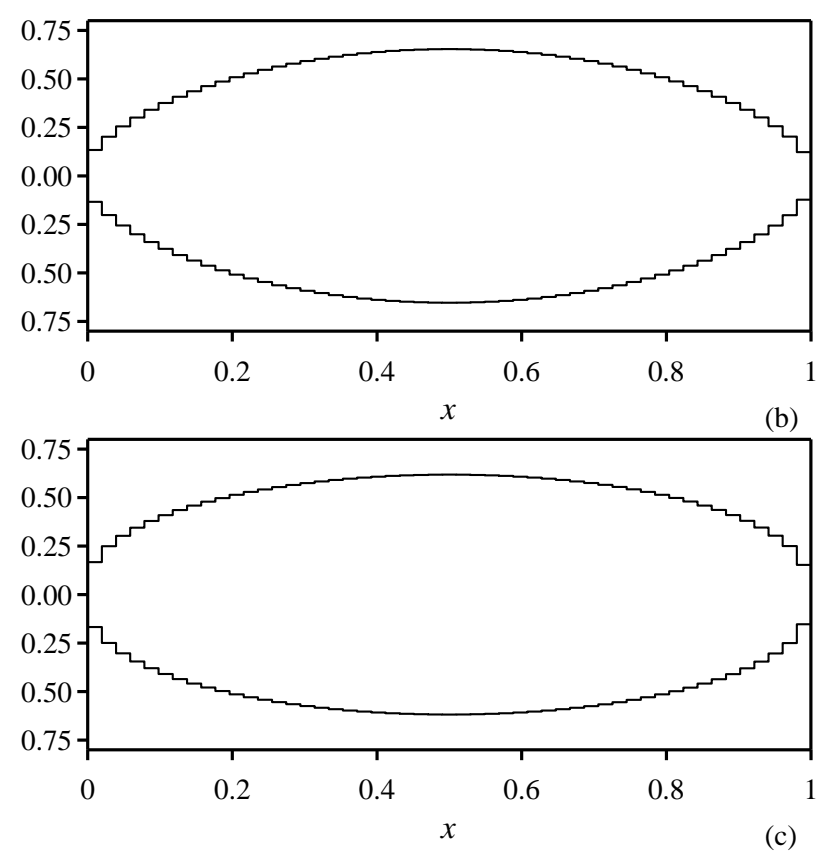

Figure 1. Optimal columns under compression (a) $n=1$, (b) $n=2$, (c) $n=3$.

\section{Conclusions}

The unimodal optimality conditions for axially compressed shear deformable columns are formulated and the problems defined are numerically solved using an iterative procedure based on finite elements.

It is shown that when the shear deformation is taken into account during optimization, the area does not vanish at points where the bending moment is zero. Also, the iterative scheme used in solving the classical column optimization problem is used here efficiently for a similar problem. However, the optimal buckling loads calculated here are found out to be very close to the optimal buckling loads calculated according to the Euler theory for most of the cases.

For shear deformable columns, only unimodal optimality conditions are formulated, but extension to the bimodal case is possible.

\section{Acknowledgement}

The author is grateful to Prof. Sarp Adali for his generous support during this research study.

\section{References}

[1]. C.M. Wang, C.Y. Wang, J.N. Reddy, 'Exact Solutions for buckling of structural members', CRC Press, Florida, USA, 2005.

[2]. M.J. Maurizi, P.M. Belles, Comments on 'The effect of shear deformations on the critical buckling of columns'. Journal of Sound and Vibration, 185:5, 908-909, 1995.

[3]. J.B. Kosmatka, An improved two-node finite element for stability and natural frequencies of axial-loaded timoshenko beams. Computers \& Structures, 57:1, 141-149, 1995.

[4]. J.R. Banarjee, F.W. Williams, The effect of shear deformation on the critical buckling of columns. Journal of Sound and Vibration, 174:5, 607-616, 1994.

[5]. C.M. Wang, R.P.S. Han, A further note on the effect of shear deformation on the critical buckling of columns. Journal of Sound and Vibration, 185:5, 895-896, 1995.

[6]. M. Avcar, Elastic buckling of steel columns under axial compression. American journal of civil engineering, 2(3), 102-108, 2014.

[7]. Cagdas, I. U., \& Adali, S. Optimization of clamped columns under distributed axial load and subject to stress constraints. Engineering Optimization, 39(4), 453-469, 2007.

[8]. Adali, S., \& Cagdas, I. U. Optimal design of simply supported columns subject to distributed axial load and stress constraint. Optimal Control Applications and Methods, 30(5), 505-520, 2009.

[9]. Cagdas, I. U., \& Adali, S. Optimal shapes of clamped-simply supported columns under distributed axial load and stress constraint. Engineering Optimization, 45(2), 123-139, 2013.

[10]. H. Ziegler, Arguments for and against Engesser's buckling formulas. Ingenieur-Archiv, 52, 105-113, 1982.

[11]. Haichang Hu, Variational Principles of Theory of Elasticity with Applications. Gordon and Breach, Science Publishers Inc., NY, 1984.

[12]. Hinton E, Owen D.P., Finite element programming. AW Billitt, Droitwich, 1977. 